\title{
Supporting Information for \\ Palladium-Catalyzed Coupling of Thiol Esters with Aryl and Primary and Secondary Alkyl Organoindium Reagents
}

Bryan W. Fausett and Lanny S. Liebeskind*

Emory University, Department of Chemistry

1515 Dickey Drive, Atlanta Georgia 30322

\section{General Experimental Methods}

Starting Materials

4-Methyl-thiobenzoic acid $S$-(4-chlorophenyl) ester, 1

S-2

4-Bromo-thiobenzoic acid $S$-(4-chlorophenyl) ester, 3

S-2

Cyclohexanecarbothioic acid $S$-(4-chlorophenyl) ester, 5

Representative Preparation of Organoindium Compounds

Triphenylindium

$t$-Butyl $(c \text {-pentyl })_{2}$ indium

Cross Coupling Products

4-Methyl-benzophenone, 2a

4-Methoxy-4'-methylbenzophenone, $2 \mathrm{~b}$

S-4

4-Fluoro-4'-methylbenzophenone, $2 \mathrm{c}$

4'-Methylvalerophenone, 2d

S-5

2-Methyl-1-p-tolyl-1-butanone, 2e

S-5

Cyclopentyl $p$-tolyl ketone, $2 \mathrm{f}$

Cyclohexyl $p$-tolyl ketone, 2g

2,4'-Dimethyl-benzophenone, $2 \mathrm{~h}$

2-Methoxy-4'-methylbenzophenone, $2 \mathrm{i}$

4-Bromo-4'-methoxybenzophenone, 4

${ }^{13}$ C NMR Spectra

\section{General Experimental Methods}

All cross coupling reactions were performed under an atmosphere of dry $\mathrm{N}_{2}$ or Ar. THF

was dried over $4 \AA$ molecular sieves and titrated for water level with a Fisher Coulomatic

K-F titrater and purged with dry $\mathrm{N}_{2}$ or Ar before use. $\mathrm{Et}_{3} \mathrm{~N}$ was dried over $\mathrm{KOH}$ pellets. 
${ }^{1} \mathrm{H}$ NMR and ${ }^{13} \mathrm{C}$ NMR spectra were taken at room temperature in $\mathrm{CDCl}_{3}$ and internally referenced to $\mathrm{CDCl}_{3}(7.26 \mathrm{ppm}, 77.23 \mathrm{ppm})$. Data are reported in the following order: chemical shifts are given $(\delta)$, multiplicities are indicated: br (broadened), s (singlet), d (doublet), t (triplet), q (quartet), hex (hextet), hept (heptext), m (multiplet), exch (exchangeable), app (apparent).

\section{Starting Materials}

4-Methylthiobenzoic acid $S$-(4-chlorophenyl) ester, 1. p-Chlorothiophenol (50 mmol, $7.23 \mathrm{~g}$ ) and $p$-toluoyl chloride (50 mmol, $7.73 \mathrm{~g}$ ) were added to $115 \mathrm{~mL}$ THF and cooled in an ice bath. To this solution triethyl amine (100 mmol, $10.12 \mathrm{~g}$ ) was added drop-wise. The reaction was allowed to warm to room temperature and stirred overnight. Diethyl ether $(100 \mathrm{~mL})$ was added and the resulting precipitated salts were filtered off. The solvent was then evaporated and the product was recrystallized from acetone giving a white solid (9.48 g, 72\% yield). m.p.: $119-120{ }^{\circ} \mathrm{C} .{ }^{1} \mathrm{H}$ NMR (600 MHz, $\left.\mathrm{CDCl}_{3}\right): \delta 7.91$ $(\mathrm{d}, J=8.4,2 \mathrm{H}), 7.43(\mathrm{~m}, 4 \mathrm{H}), 7.29(\mathrm{~d}, J=9.0,2 \mathrm{H}), 2.44(\mathrm{~s}, 3 \mathrm{H}) .{ }^{13} \mathrm{C} \mathrm{NMR}(150 \mathrm{MHz}$, $\left.\mathrm{CDCl}_{3}\right) \delta 189.4,145.1,136.6,136.1,134.0,129.7,127.8,126.2,22.0$. IR (neat): 1671

(s). Anal. Calcd for $\mathrm{C}_{14} \mathrm{H}_{11} \mathrm{~S}$ : C, 64.00; H, 4.22; S, 12.20\%. Found: C, 64.04; H, 4.42; S, $12.15 \%$.

4-Bromo-thiobenzoic acid $S$-(4-chlorophenyl) ester, 3. p-Chlorothiophenol (10 mmol, $1.45 \mathrm{~g}$ ) and 4-bromobenzoyl chloride (10 mmol, $2.19 \mathrm{~g}$ ) were added to $30 \mathrm{~mL}$ THF and cooled in an ice bath. To this solution triethyl amine (20 mmol, $2.02 \mathrm{~g}$ ) was added dropwise. The reaction was allowed to warm to room temperature and stirred overnight. Diethyl ether $(20 \mathrm{~mL})$ was added and the resultant precipitated salts were filtered off. The solvent was then evaporated and the product recrystallized from acetone giving a 
white solid (2.36 g, 72\% yield). m.p.: $145-146{ }^{\circ} \mathrm{C} .{ }^{1} \mathrm{H}$ NMR (600 MHz, $\left.\mathrm{CDCl}_{3}\right): \delta 7.88$ $(\mathrm{d}, J=8.4,2 \mathrm{H}), 7.65(\mathrm{~d}, J=8.4,2 \mathrm{H}), 7.44(\mathrm{~s}, 4 \mathrm{H}) .{ }^{13} \mathrm{C} \mathrm{NMR}\left(150 \mathrm{MHz}, \mathrm{CDCl}_{3}\right) \delta$ 189.0, 136.5, 136.4, 135.4, 132.4, 129.8, 129.23, 129.16, 125.6. IR (neat): 1675 (s). Anal. Calcd for $\mathrm{C}_{13} \mathrm{H}_{8} \mathrm{~S}: \mathrm{C}, 47.66 ; \mathrm{H}, 2.46 ; \mathrm{S}, 9.79 \%$. Found: $\mathrm{C}, 47.42 ; \mathrm{H}, 2.55 ; \mathrm{S}$, $9.80 \%$.

Cyclohexanecarbothioic acid $S$-(4-chlorophenyl) ester, 5. $p$-Chlorothiophenol (10 mmol, $1.45 \mathrm{~g})$ and cyclohexanecarbonyl chloride $(10 \mathrm{mmol}, 1.47 \mathrm{~g})$ were added to $30 \mathrm{~mL}$ THF and cooled in an ice bath. To this solution triethyl amine $(20 \mathrm{mmol}, 2.02 \mathrm{~g})$ was added drop-wise. The reaction was allowed to warm to room temperature and stirred overnight. Diethyl ether $(20 \mathrm{~mL})$ was added and the resultant precipitated salts were filtered off. The solvent was then evaporated and the product recrystallized from acetone giving a white solid (2.33 g, $91 \%$ yield). m.p.: $34-35{ }^{\circ} \mathrm{C} .{ }^{1} \mathrm{H}$ NMR (400 MHz, $\left.\mathrm{CDCl}_{3}\right): \delta$ 7.34 (m, $4 \mathrm{H}), 2.60$ (m, $1 \mathrm{H}), 2.01-1.27$ (m, $10 \mathrm{H}) .{ }^{13} \mathrm{C} \mathrm{NMR}\left(100 \mathrm{MHz}, \mathrm{CDCl}_{3}\right) \delta 200.3$, 136.0, 135.7, 129.5, 126.6, 52.7, 29.6, 25.7, 25.6. IR (neat): 1702 (s). Anal. Calcd for $\mathrm{C}_{13} \mathrm{H}_{15} \mathrm{~S}: \mathrm{C}, 61.28 ; \mathrm{H}, 5.93 ; \mathrm{S}, 12.59 \%$. Found: C, 61.02; H, 5.98; S, $12.40 \%$.

\section{Representative Preparation of Organoindium Compounds}

Triphenylindium: Indium(III) chloride $(0.2 \mathrm{mmol}, 44 \mathrm{mg})$ was placed in a flask with a vacuum attachment and stirring bar. This was heated with a heat gun while stirring under vacuum for several minutes to ensure dryness. After heating, the flask was filled with Ar and then re-evacuated; this was repeated 3 times before filling the flask a final time with Ar. Next, THF $(1.5 \mathrm{~mL})$ was added via syringe. Once the indium(III) chloride was dissolved phenyl $\mathrm{MgCl}(0.6 \mathrm{mmol})$ was added drop-wise and allowed to stir for $0.5 \mathrm{~h}$. This was added directly to the coupling reaction without isolation. 
tert-Butyl(cyclopentyl) $)_{2}$ indium: Indium(III) chloride $(0.37 \mathrm{mmol}, 83 \mathrm{mg})$ was placed in a flask with a vacuum attachment and stirring bar. This was heated with a heat gun while stirring under vacuum for several minutes to ensure dryness. After heating the flask was filled with Ar and then re-evacuated; this was repeated 3 times before filling the flask a final time with Ar. Next, THF (1.5 mL dry/degassed) was added via syringe. Once the indium(III) chloride was dissolved tert-ButylMgCl (0.37 mmol, 1.9 $\mathrm{M}$ in diethyl ether, $0.20 \mathrm{~mL}$ ) was added drop-wise and allowed to stir for $0.5 \mathrm{~h}$ giving a slightly milky reaction mixture. To this was added cyclopentyl $\mathrm{MgCl}(0.75 \mathrm{~mol}, 1.96 \mathrm{M}$ in diethyl ether, $0.39 \mathrm{~mL}$ ) drop-wise and the resultant solution was stirred another $0.5 \mathrm{~h}$ to give a solution of tert-butyl(cyclopentyl $)_{2}$ indium. This was added directly to the coupling reaction.

\section{Cross Coupling Products}

4-Methylbenzophenone, $2 \mathrm{a}^{1}{ }^{4-M e t h y l t h i o b e n z o i c ~ a c i d ~} S$-(4-chlorophenyl) ester $(0.50$ mmol, $131 \mathrm{mg})$ was added with $\mathrm{Pd}(\mathrm{MeCN})_{2} \mathrm{Cl}_{2}(5 \mathrm{~mol} \%, 6.5 \mathrm{mg})$ to a flask that was subsequently flushed with Ar. THF $(0.5 \mathrm{~mL})$ was added to the flask immediately followed by the triphenyl indium solution. Finally, this was placed in an oil bath at $55{ }^{\circ} \mathrm{C}$ and stirred for $\sim 16 \mathrm{~h}$. The reaction was worked up, filtered through a silica plug and evaporated. After preparative plate chromatography $\left(1: 1 \mathrm{CH}_{2} \mathrm{Cl}_{2} /\right.$ hexanes $)$ a colorless oil was obtained (87 mg, 89\% yield). ${ }^{1} \mathrm{H} \mathrm{NMR}\left(600 \mathrm{MHz}, \mathrm{CDCl}_{3}\right): \delta 7.77(\mathrm{~d}, J=7.2,2 \mathrm{H})$, $7.71(\mathrm{~d}, J=7.8,2 \mathrm{H}), 7.56(\mathrm{~m}, 1 \mathrm{H}), 7.46(\mathrm{~m}, 2 \mathrm{H}), 7.27(\mathrm{~d}, J=8.4,2 \mathrm{H}), 2.42(\mathrm{~s}, 3 \mathrm{H})$.

${ }^{13} \mathrm{C} \mathrm{NMR}\left(150 \mathrm{MHz}, \mathrm{CDCl}_{3}\right) \delta 196.7,143.4,138.1,135.0,132.3,130.5,130.1,129.1$, 128.4, 21.8. IR (neat): 1656 (s).

4-Methoxy-4'-methylbenzophenone, 2b. ${ }^{2}$ 4-Methylthiobenzoic acid $S$-(4-chlorophenyl) ester $(0.50 \mathrm{mmol}, 131 \mathrm{mg}), \operatorname{Pd}\left(\mathrm{MeCN}_{2} \mathrm{Cl}_{2}(5 \mathrm{~mol} \%, 6.5 \mathrm{mg})\right.$, and (tert-Bu$) \operatorname{In}(p-$ 
methoxyphenyl $)_{2}(0.3 \mathrm{mmol})$ gave 4-methoxy-4'-methylbenzophenone (81 $\left.\mathrm{mg}, 72 \%\right)$ as a white solid. m.p.: $88-89{ }^{\circ} \mathrm{C} .{ }^{1} \mathrm{H}$ NMR $\left(600 \mathrm{MHz}, \mathrm{CDCl}_{3}\right): \delta 7.80(\mathrm{~d}, J=9.0,2 \mathrm{H}), 7.66$ $(\mathrm{d}, J=8.4,2 \mathrm{H}), 7.26(\mathrm{~d}, J=8.4,2 \mathrm{H}), 6.94(\mathrm{~d}, J=8.4,2 \mathrm{H}), 2.42(\mathrm{~s}, 3 \mathrm{H}) .{ }^{13} \mathrm{C} \mathrm{NMR}$ $\left(150 \mathrm{MHz}, \mathrm{CDCl}_{3}\right) \delta 195.6,163.2,142.8,135.7,132.6,130.7,130.2,129.1,113.7,55.7$, 21.8. IR (neat): 1652 (s).

4-Fluoro-4'-methylbenzophenone, $2 c^{3}{ }^{3}$ 4-Methylthiobenzoic acid $S$-(4-chlorophenyl) ester $(0.50 \mathrm{mmol}, 131 \mathrm{mg}), \operatorname{Pd}\left(\mathrm{MeCN}_{2} \mathrm{Cl}_{2}(5 \mathrm{~mol} \%, 6.5 \mathrm{mg})\right.$, and (tert-Bu$) \operatorname{In}(p$ fluorophenyl $)_{2}(0.3 \mathrm{mmol})$ gave 4-fluoro-4'-methylbenzophenone $(91 \mathrm{mg}, 85 \%)$ as a white solid. m.p.: $96-97{ }^{\circ} \mathrm{C} .{ }^{1} \mathrm{H}$ NMR $\left(600 \mathrm{MHz}, \mathrm{CDCl}_{3}\right): \delta 7.83(\mathrm{~m}, 2 \mathrm{H}), 7.69(\mathrm{~d}, J=$ 7.8, $2 \mathrm{H}), 7.29$ (d, J = 7.8, $2 \mathrm{H}), 7.15(\mathrm{~m}, 2 \mathrm{H}), 2.44(\mathrm{~s}, 3 \mathrm{H}) .{ }^{13} \mathrm{C} \mathrm{NMR}(150 \mathrm{MHz}$, $\left.\mathrm{CDCl}_{3}\right) \delta 195.2,166.3,164.6,143.5,135.0,134.3,132.75,132.68,130.3,129.3,115.6$, 115.5, 22.8. IR (neat): 1648 (s).

4'-Methylvalerophenone, $2 \mathbf{d}^{4}{ }^{4}$ 4-Methylthiobenzoic acid $S$-(4-chlorophenyl) ester (0.50 mmol, $131 \mathrm{mg}), \mathrm{Pd}\left(\mathrm{MeCN}_{2} \mathrm{Cl}_{2}(5 \mathrm{~mol} \%, 6.5 \mathrm{mg})\right.$, and (tert-Bu) $\operatorname{In}(n \text {-butyl })_{2}(0.3 \mathrm{mmol})$ gave 4'-methylvalerophenone $(73 \mathrm{mg}, 83 \%)$ as a clear yellow oil. ${ }^{1} \mathrm{H}$ NMR $(600 \mathrm{MHz}$, $\left.\mathrm{CDCl}_{3}\right): \delta 7.84(\mathrm{~d}, J=8.4,2 \mathrm{H}), 7.22(\mathrm{~d}, J=7.8,2 \mathrm{H}), 2.91(\mathrm{t}, J=7.2,2 \mathrm{H}), 2.38(\mathrm{~s}, 3 \mathrm{H})$, $1.69(\mathrm{~m}, 2 \mathrm{H}), 1.38(\mathrm{~m}, 2 \mathrm{H}), 0.93(\mathrm{t}, J=7.2,3 \mathrm{H}) .{ }^{13} \mathrm{C} \mathrm{NMR}\left(150 \mathrm{MHz}, \mathrm{CDCl}_{3}\right) \delta 200.4$, $143.7,134.8,129.4,128.3,38.4,26.8,22.6,21.7,14.1$. IR (neat): 1648 (s).

2-Methyl-1-p-tolyl-1-butanone, 2 e $^{5}$ 4-Methylthiobenzoic acid $S$-(4-chlorophenyl) ester $(0.50 \mathrm{mmol}, 131 \mathrm{mg}), \mathrm{Pd}(\mathrm{MeCN})_{2} \mathrm{Cl}_{2}(5 \mathrm{~mol} \%, 6.5 \mathrm{mg})$, and (tert-Bu) $\operatorname{In}(\text { sec-butyl })_{2}$ (0.375 mmol) gave 2-methyl-1-p-tolyl-1-butanone $(73 \mathrm{mg}, 83 \%)$ as a clear yellow oil. ${ }^{1} \mathrm{H}$ NMR (600 MHz, $\left.\mathrm{CDCl}_{3}\right): \delta 7.85(\mathrm{~d}, J=7.8,2 \mathrm{H}), 7.24(\mathrm{~d}, J=7.8,2 \mathrm{H}), 3.36(\mathrm{~m}, 1 \mathrm{H})$, $2.39(\mathrm{~s}, 3 \mathrm{H}), 1.81(\mathrm{~m}, 1 \mathrm{H}), 1.47(\mathrm{~m}, 1 \mathrm{H}), 1.16(\mathrm{~d}, J=6.6,3 \mathrm{H}), 0.89$ (t, $J=7.5,3 \mathrm{H})$. 
${ }^{13} \mathrm{C}$ NMR $\left(150 \mathrm{MHz}, \mathrm{CDCl}_{3}\right) \delta 204.4,143.7,134.5,129.5,128.6,42.1,26.9,21.8,17.0$, 12.0. IR (neat): 1679 (s).

Cyclopentyl $p$-tolyl ketone, 2f. ${ }^{6}$ 4-Methylthiobenzoic acid $S$-(4-chlorophenyl) ester (0.50 mmol, $131 \mathrm{mg}), \mathrm{Pd}(\mathrm{MeCN})_{2} \mathrm{Cl}_{2}(5 \mathrm{~mol} \%, 6.5 \mathrm{mg})$, and (tert-Bu) $\operatorname{In}(\text { cyc-pentyl })_{2}$ (0.375 mmol) gave cyclopentyl $p$-tolyl ketone $(75 \mathrm{mg}, 80 \%)$ as a clear yellow oil. ${ }^{1} \mathrm{H}$ NMR (300 MHz, $\left.\mathrm{CDCl}_{3}\right): \delta 7.87(\mathrm{~d}, J=8.1,2 \mathrm{H}), 7.25(\mathrm{~d}, J=8.1,2 \mathrm{H}), 3.69(\mathrm{~m}, 1 \mathrm{H})$, 2.40 (s, $3 \mathrm{H}), 1.90$ (m, $4 \mathrm{H}), 1.68(\mathrm{~m}, 4 \mathrm{H}) .{ }^{13} \mathrm{C} \mathrm{NMR}\left(100 \mathrm{MHz}, \mathrm{CDCl}_{3}\right) \delta$ 204.3, 143.7, 134.5, 129.5, 128.5, 42.1, 26.9, 21.7, 17.0, 12.0. IR (neat): 1679 (s).

Cyclohexyl $\boldsymbol{p}$-tolyl ketone, 2g. ${ }^{7}$ 4-Methylthiobenzoic acid $S$-(4-chlorophenyl) ester (0.50 $\mathrm{mmol}, 131 \mathrm{mg}), \mathrm{Pd}(\mathrm{MeCN})_{2} \mathrm{Cl}_{2}(5 \mathrm{~mol} \%, 6.5 \mathrm{mg})$, and (tert-Bu) $\operatorname{In}(\text { cyc-hexyl })_{2}(0.375$ mmol) gave cyclohexyl $p$-tolyl ketone (96 mg, $95 \%$ ) as a white solid. m.p.: $61-63{ }^{\circ} \mathrm{C}$. ${ }^{1} \mathrm{H}$ NMR (400 MHz, $\left.\mathrm{CDCl}_{3}\right): \delta 7.83(\mathrm{~d}, J=6.8,2 \mathrm{H}), 7.23(\mathrm{~d}, J=8.4,2 \mathrm{H}), 3.22(\mathrm{~m}, 1$ H), 2.38 (s, $3 \mathrm{H}), 1.84(\mathrm{~m}, 4 \mathrm{H}), 1.74(\mathrm{~m}, 1 \mathrm{H}), 1.49-1.23(\mathrm{~m}, 5 \mathrm{H}) .{ }^{13} \mathrm{C} \mathrm{NMR}(100 \mathrm{MHz}$, $\left.\mathrm{CDCl}_{3}\right) \delta 203.7,143.6,133.9,129.4,128.5,45.7,29.6,26.1,26.0,21.7 . \quad$ IR (neat): 1675 (s). Alternatively, cyclohexyl $p$-tolyl ketone $\mathbf{2 g}$ can be prepared by the coupling of cyclohexanecarbothioic acid $S$-(4-chlorophenyl) ester with (tert-Bu) $\operatorname{In}(p$-tolyl) 2 : Cyclohexanecarbothioic acid $S$-(4-chlorophenyl) ester (0.50 mmol, $127 \mathrm{mg})$, $\operatorname{Pd}(\mathrm{MeCN})_{2} \mathrm{Cl}_{2}(5 \mathrm{~mol} \%, 6.5 \mathrm{mg})$, and (tert-Bu) $\operatorname{In}(p \text {-tolyl })_{2}(0.375 \mathrm{mmol})$ gave cyclohexyl $p$-tolyl ketone $(53 \mathrm{mg}, 91 \%)$ as a white solid. m.p.: $61-63{ }^{\circ} \mathrm{C}$. 2,4'-Dimethylbenzophenone, $2 \mathbf{h}^{\mathbf{8}}{ }^{4}$-Methylthiobenzoic acid $S$-(4-chlorophenyl) ester (0.50 mmol, $131 \mathrm{mg}), \mathrm{Pd}(\mathrm{MeCN})_{2} \mathrm{Cl}_{2}(5 \mathrm{~mol} \%, 6.5 \mathrm{mg})$, and (tert-Bu)In(o-tolyl $)_{2}(0.3$ mmol) gave 2,4'-dimethylbenzophenone (58 mg, 55\%) as a clear yellow oil. ${ }^{1} \mathrm{H}$ NMR (400 MHz, $\left.\mathrm{CDCl}_{3}\right): \delta 7.70(\mathrm{~d}, J=8.0,2 \mathrm{H}), 7.37(\mathrm{~m}, 1 \mathrm{H}), 7.30-7.21(\mathrm{~m}, 5 \mathrm{H}), 2.41(\mathrm{~s}$, 
3H), $2.31(\mathrm{~s}, 3 \mathrm{H}) .{ }^{13} \mathrm{C} \mathrm{NMR}\left(100 \mathrm{MHz}, \mathrm{CDCl}_{3}\right) \delta 198.6,144.3,139.2,136.7,135.4$, 131.1, 130.5, 130.2, 129.4, 128.5, 125.4, 21.9. 20.1. IR (neat): 1660 (s).

2-Methoxy-4'-methylbenzophenone, 2i. ${ }^{9}$ 4-Methylthiobenzoic acid $S$-(4-chlorophenyl) ester $(0.50 \mathrm{mmol}, 131 \mathrm{mg}), \mathrm{Pd}(\mathrm{MeCN})_{2} \mathrm{Cl}_{2}(5 \mathrm{~mol} \%, 6.5 \mathrm{mg})$, and $($ tert-Bu$) \operatorname{In}(o-$ methoxyphenyl) (0.3 mmol) gave 2-methoxy-4'-methylbenzophenone (68 $\mathrm{mg}, 60 \%)$ as a white solid. m.p.: $64-65{ }^{\circ} \mathrm{C} .{ }^{1} \mathrm{H}$ NMR $\left(400 \mathrm{MHz}, \mathrm{CDCl}_{3}\right): \delta 7.72(\mathrm{~d}, J=8.4,2 \mathrm{H}), 7.45$ $(\mathrm{dt}, J=8.0, J=1.8,1 \mathrm{H}), 7.33(\mathrm{dd}, J=7.2, J=1.6,1 \mathrm{H}), 7.23(\mathrm{~d}, J=8.0,2 \mathrm{H}), 7.03(\mathrm{t}, J$ $=7.6,1 \mathrm{H}), 6.99(\mathrm{~d}, J=8.8,1 \mathrm{H}), 3.73(\mathrm{~s}, 3 \mathrm{H}), 2.41(\mathrm{~s}, 3 \mathrm{H}) .{ }^{13} \mathrm{C} \mathrm{NMR}(100 \mathrm{MHz}$, $\left.\mathrm{CDCl}_{3}\right) \delta 196.4,157.4,144.0,135.4,131.8,130.2,129.6,129.3,129.1,120.6,111.6$, 55.8, 21.9. IR (neat): 1660 (s).

4-Bromo-4'-methoxybenzophenone, 4. $^{10}$ 4-Bromothiobenzoic acid $S$-(4-chlorophenyl) ester $(0.50 \mathrm{mmol}, 131 \mathrm{mg}), \mathrm{Pd}(\mathrm{MeCN})_{2} \mathrm{Cl}_{2}(5 \mathrm{~mol} \%, 6.5 \mathrm{mg})$, and $($ tert $-\mathrm{Bu}) \operatorname{In}(p-$ methoxyphenyl $)_{2}(0.30 \mathrm{mmol})$ gave 4-bromo-4'-methoxybenzophenone $(138 \mathrm{mg}, 95 \%)$ as a yellow solid. m.p.: $151-152{ }^{\circ} \mathrm{C} .{ }^{1} \mathrm{H} \mathrm{NMR}\left(400 \mathrm{MHz}, \mathrm{CDCl}_{3}\right): \delta 7.79(\mathrm{~d}, J=8.8,2 \mathrm{H})$, $7.62(\mathrm{~m}, 4 \mathrm{H}), 6.97(\mathrm{~d}, J=8.8,2 \mathrm{H}), 3.89(\mathrm{~s}, 3 \mathrm{H}) .{ }^{13} \mathrm{C} \mathrm{NMR}\left(150 \mathrm{MHz}, \mathrm{CDCl}_{3}\right) \delta$ 194.6, 163.6, 137.2, 132.7, 131.7, 131.5, 129.9, 127.0, 113.9, 55.7. IR (neat): 1640 (s).

\footnotetext{
${ }^{1}$ Molander, G. A.; Yun, C.-S.; Ribagorda, M.; Biolatto, B. J. Org. Chem., 2003, 68, 5534.

${ }^{2}$ Atkinson, G. E.; Fischer, P. M.; Chan, W. C. J. Org. Chem., 2000, 65, 5048.

${ }^{3}$ Pews et al. J. Amer. Chem. Soc., 1967, 89, 2391.

${ }^{4}$ Katritzky, A. R.; Kuzmierkiewicz, W. J. Chem. Soc. Perkin Trans. 1, 1987, 819.

${ }^{5}$ Al-Aseer, M. A.; Smith, S. G.; J. Org. Chem., 1984, 49, 2608.

${ }^{6}$ Hori, M.; Kataoka, T.; Shimizu, H.; Imai, E.; Iwamura, T.; Maeda, K. Chem. Pharm. Bull., 1986, 34, 3599.

${ }^{7}$ Kondo, T.; Akazome, M.; Tsuji, Y.; Watanabe, Y. J. Org. Chem., 1990, 55, 1286.

${ }^{8}$ Nishio, T.; Tokunaga, T.; Omote, Y. Synth. Commun., 1988, 18, 2083.

${ }^{9}$ Xia, M.; Chen, Z. J.Chem. Res. Synop., 1999, 6, 400.

${ }^{10}$ Andrus, M. B.; Ma, Y.; Zang, Y.; Song, C. Tetrahedron Lett., 2002, 42, 9137.
} 

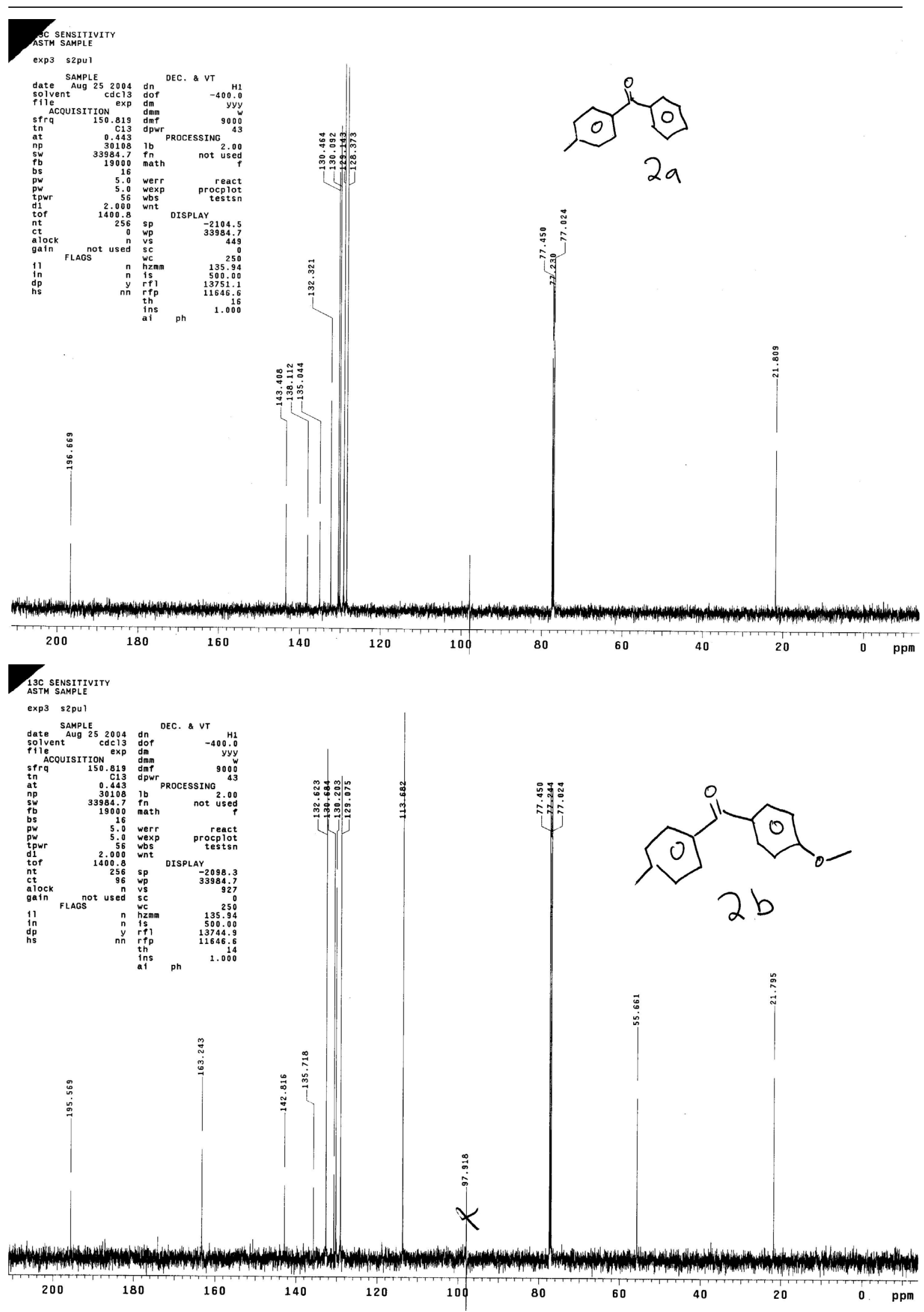


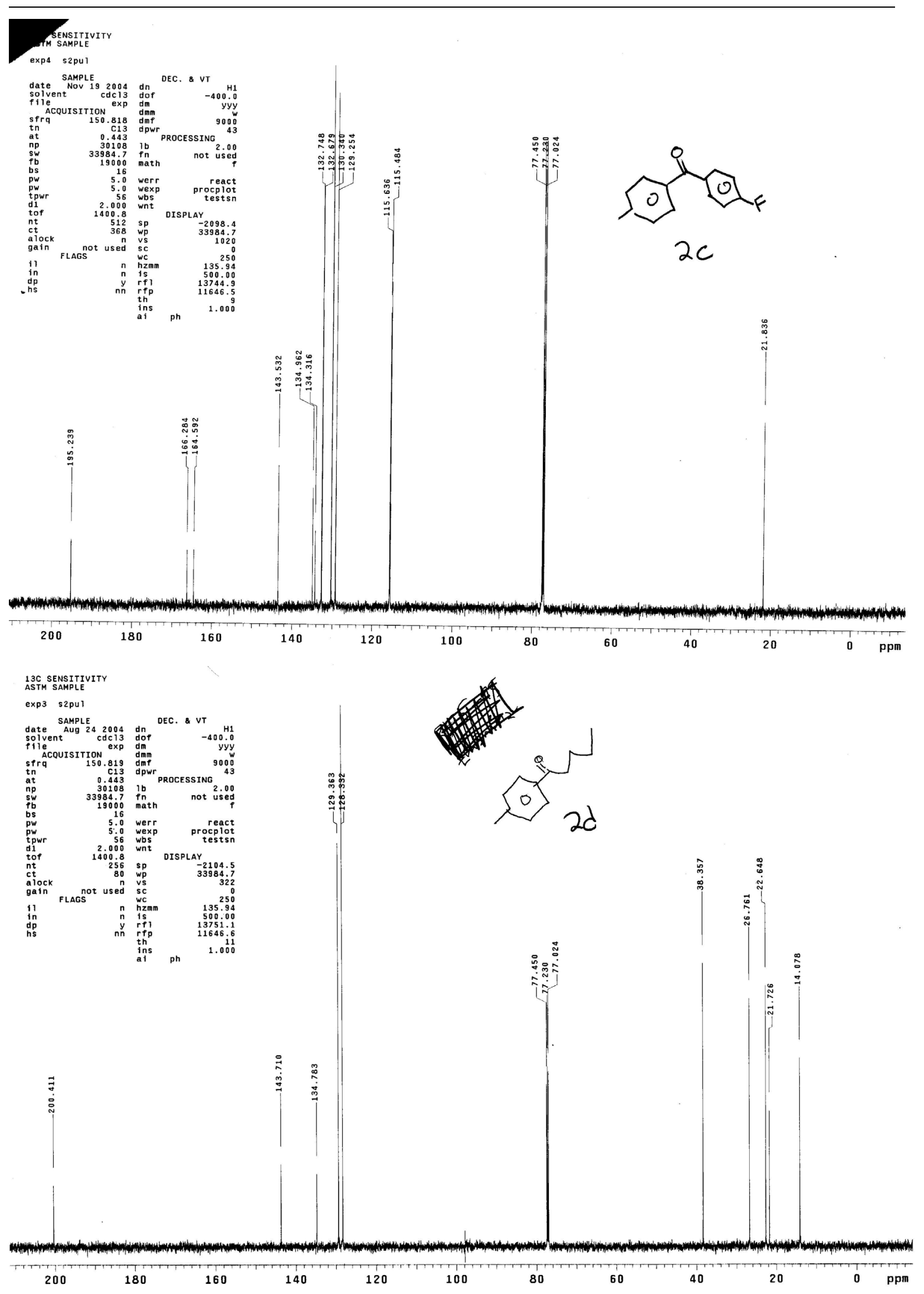




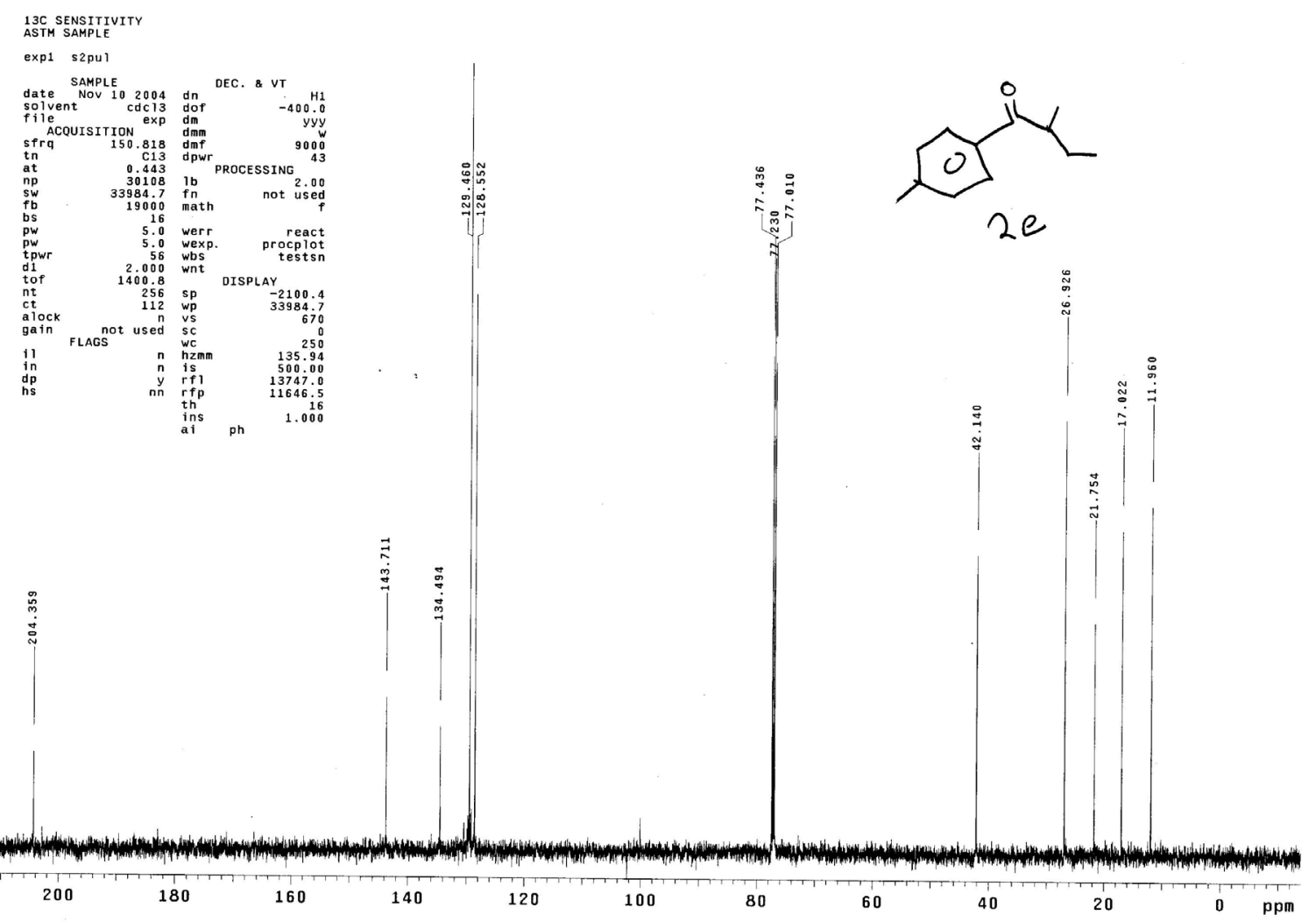

13C OBSERVE

exp1 std13c
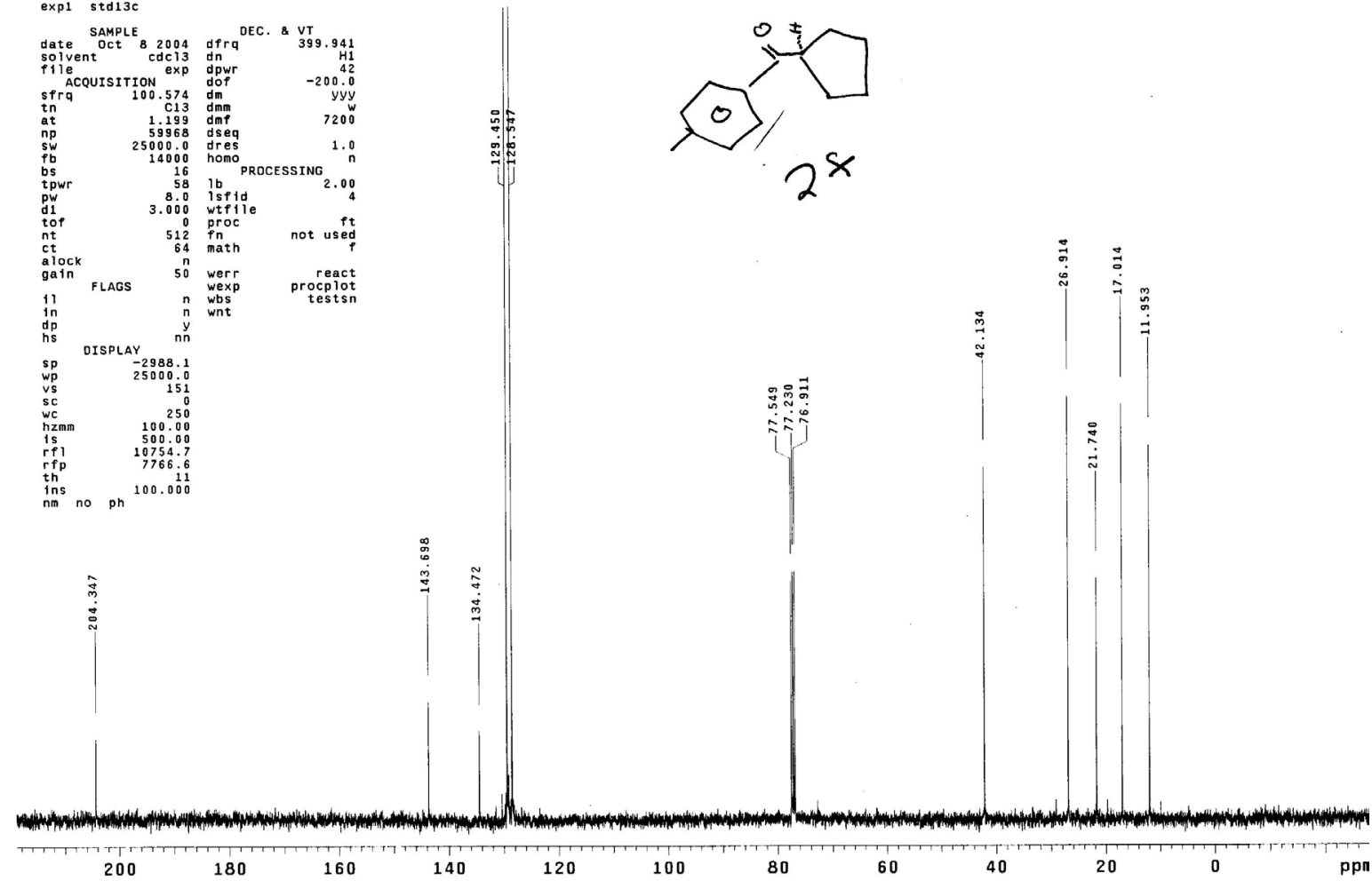
13C observe

\section{exp2 std13c}
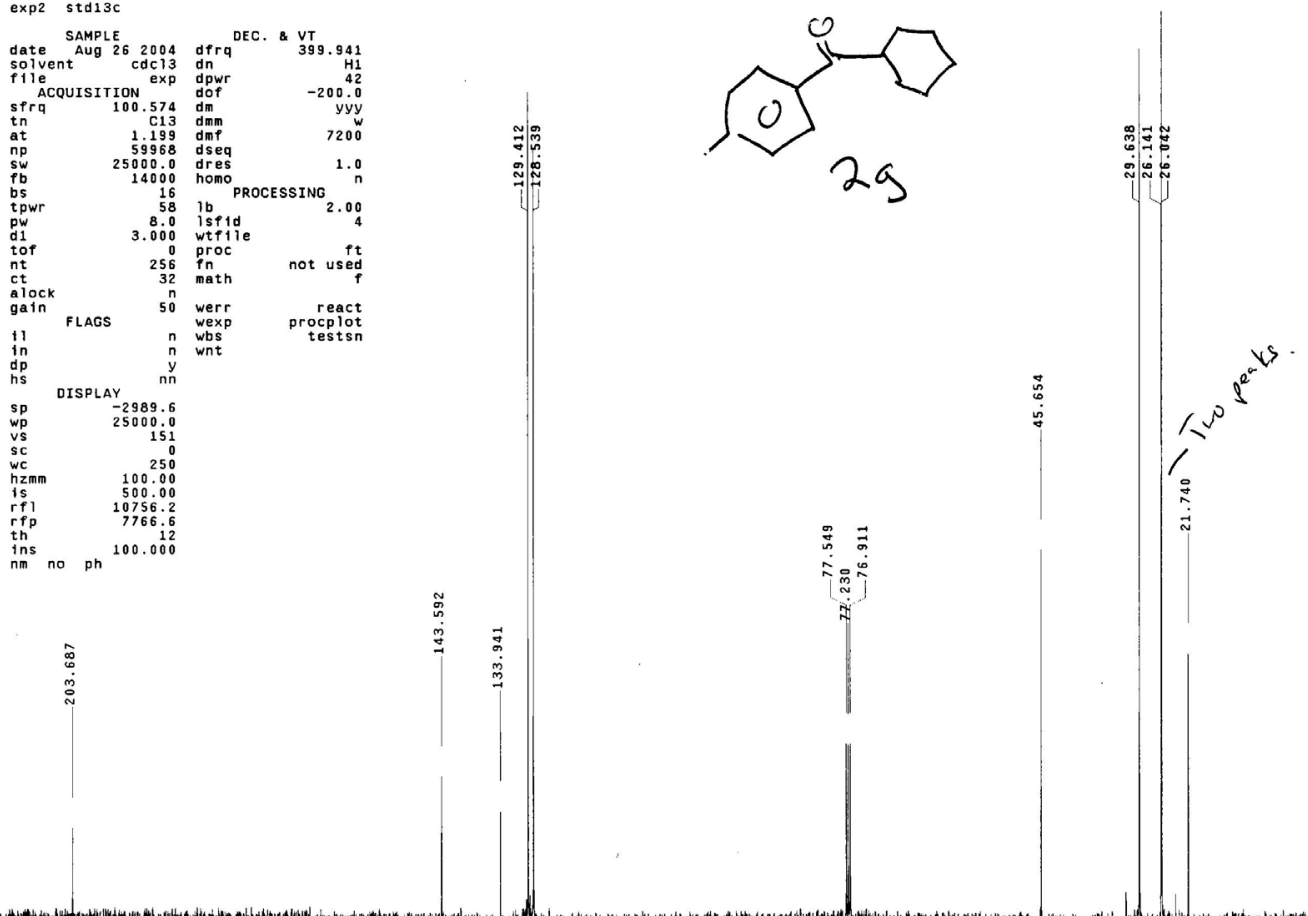

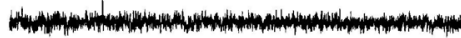

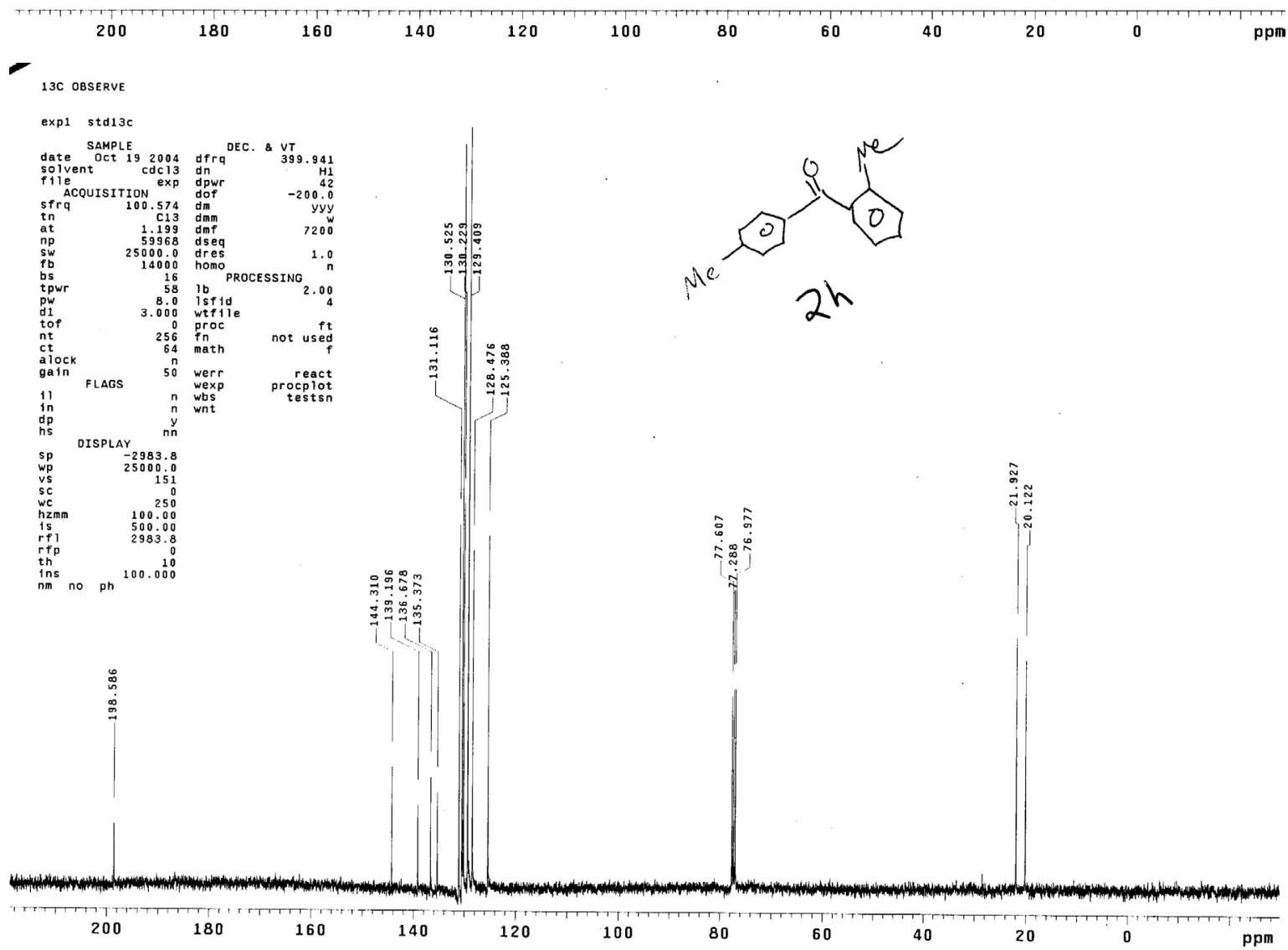




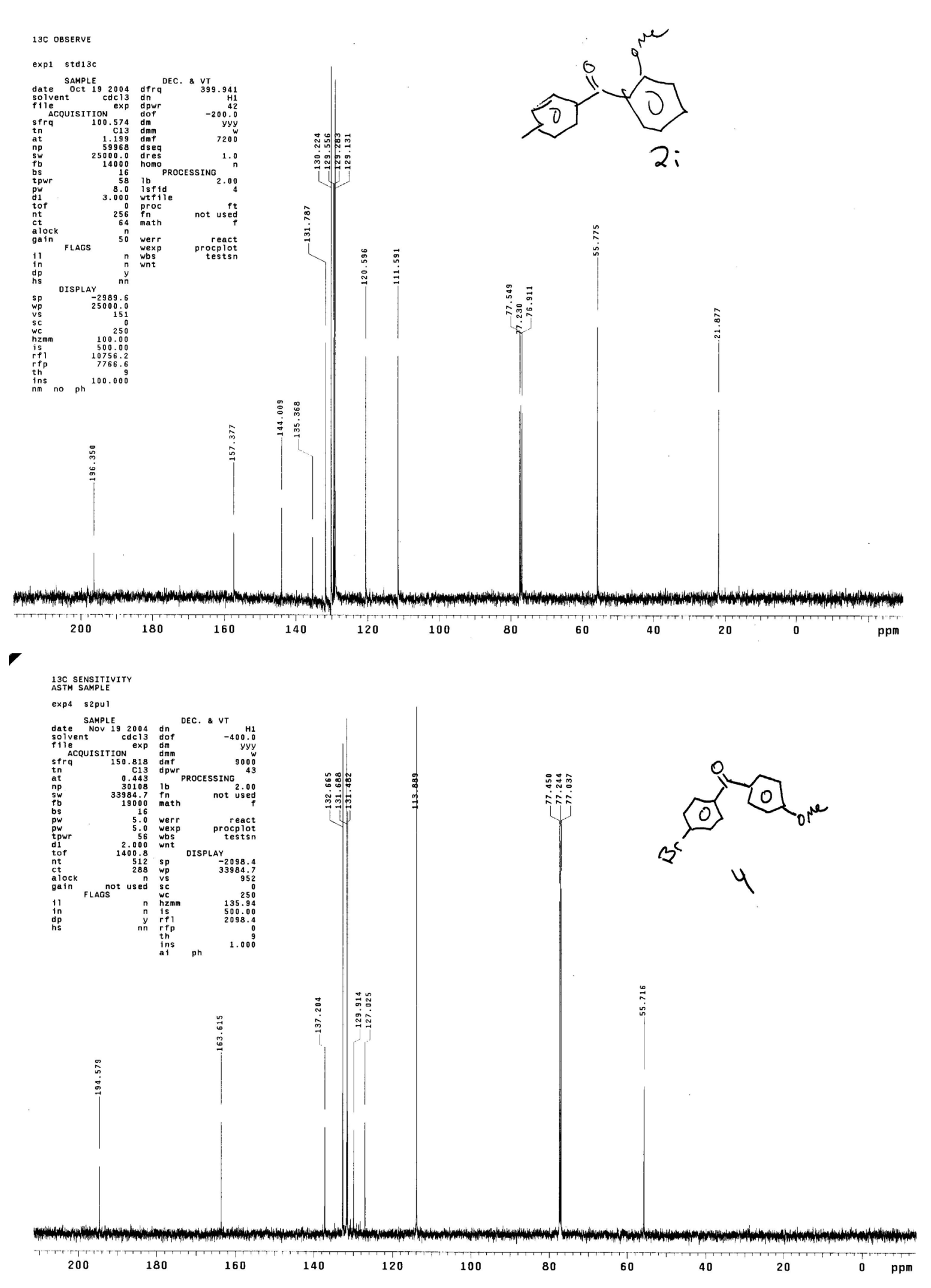

